\title{
Teaching NeuroImages: Osteolytic intraosseous meningioma causing transcalvarial herniation
}

Aurora Semerano, MD, * Antonino Giordano, MD, * Mariarosa Terreni, MD, Lina Raffaella Barzaghi, MD, Giancarlo Comi, MD, Maria Antonietta Volonté, MD, and Massimo Filippi, MD

Neurology ${ }^{\circledR}$ 2020;95:e1110-e1111. doi:10.1212/WNL.0000000000010091

Figure 1 Imaging showing a lytic lesion of right parietal bone with transcalvarial herniation

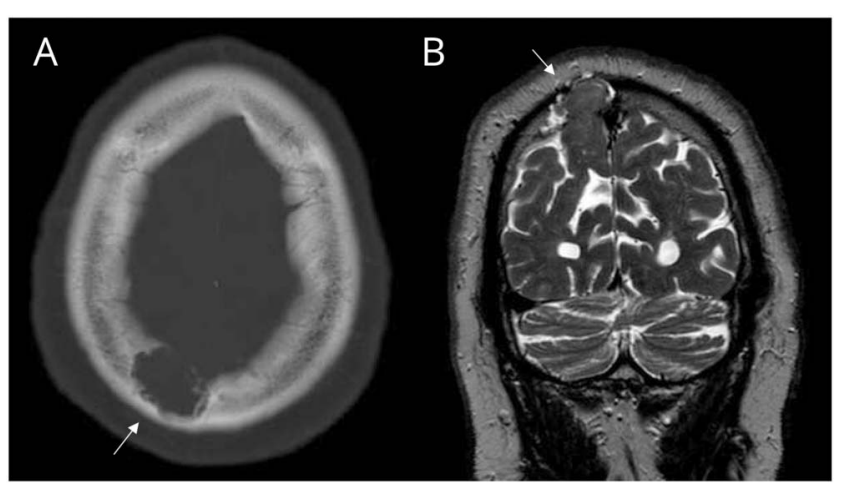

(A) Axial bone-window CT scan showing a lytic right parietal skull lesion. (B) Coronal T2-weighted MRI scan showing transcalvarial herniation.

A 69-year-old man presented for focal seizures characterized by dysarthria, confusion, left hand paresthesia, and hemifacial contractions. He had a 3-year history of episodes of epigastric burning, followed by confusion, olfactory hallucinations, and déjà vu. Head CT scan showed a lytic lesion of right parietal bone (figure 1A). MRI highlighted transcalvarial herniation of

\section{Correspondence}

Prof. Filippi

filippi.massimo@hsr.it

\section{MORE ONLINE}

$\rightarrow$ Teaching slides

links.lww.com/WNL/

B139

Figure 2 Macroscopic and microscopic features of the lesion
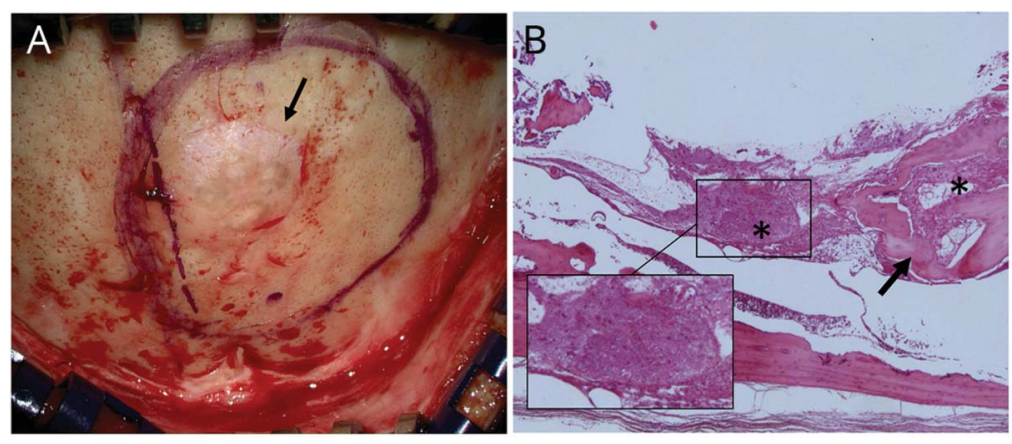

(A) Herniated brain tissue visible under a thin layer of skull. (B) Histopathologic examination revealing meningioma (asterisks) infiltrating the bone layer (arrow).

*These authors contributed equally to this work.

From the Neurology Unit (A.S., A.G., G.C., M.A.V., M.F.), Pathology Unit (M.T.), and Neurosurgery Unit (L.R.B.), IRCCS San Raffaele Scientific Institute; and Vita-Salute San Raffaele University (A.S., A.G., M.F.), Milan, Italy.

Go to Neurology.org/N for full disclosures. Funding information and disclosures deemed relevant by the authors, if any, are provided at the end of the article. 
precuneus gyrus (figure 1B), suggesting a congenital or posttraumatic skull defect or an osteolytic lesion. After surgical repair and excision of adjacent injured brain tissue, histopathology revealed osteolytic meningioma (figure 2). Intraosseous meningioma is extremely rare, may cause transcalvarial herniation by osteolysis, ${ }^{1}$ and can be revealed by histopathology.

\section{Study funding}

No targeted funding reported.

\section{Disclosure}

The authors report no disclosures relevant to the manuscript. Go to Neurology.org/N for full disclosures.

Appendix Authors

\begin{tabular}{lll}
\hline Name & Location & Contribution \\
\hline $\begin{array}{l}\text { Aurora } \\
\text { Memerano, }\end{array}$ & $\begin{array}{l}\text { IRCCS San Raffaele } \\
\text { Scientific Institute, } \\
\text { Milan, Italy }\end{array}$ & $\begin{array}{l}\text { Study concept, clinical } \\
\text { assessment, analysis and } \\
\text { interpretation of the data, } \\
\text { drafting/revising the manuscript }\end{array}$ \\
\hline $\begin{array}{l}\text { Antonino } \\
\text { Giordano, }\end{array}$ & IRCCS San Raffaele & $\begin{array}{l}\text { Study concept, clinical } \\
\text { assessment, analysis and } \\
\text { MD }\end{array}$ \\
Milan, Italy & drafting/revising the manuscript \\
& &
\end{tabular}

Appendix (continued)

\begin{tabular}{|c|c|c|}
\hline Name & Location & Contribution \\
\hline $\begin{array}{l}\text { Mariarosa } \\
\text { Terreni, MD }\end{array}$ & $\begin{array}{l}\text { IRCCS San Raffaele } \\
\text { Scientific Institute, } \\
\text { Milan, Italy }\end{array}$ & $\begin{array}{l}\text { Neuropathologic study, } \\
\text { analysis and interpretation of } \\
\text { the data, revising the } \\
\text { manuscript }\end{array}$ \\
\hline $\begin{array}{l}\text { Lina } \\
\text { Raffaella } \\
\text { Barzaghi, } \\
\text { MD }\end{array}$ & $\begin{array}{l}\text { IRCCS San Raffaele } \\
\text { Scientific Institute, } \\
\text { Milan, Italy }\end{array}$ & $\begin{array}{l}\text { Clinical assessment, analysis } \\
\text { and interpretation of the data, } \\
\text { revising the manuscript }\end{array}$ \\
\hline $\begin{array}{l}\text { Giancarlo } \\
\text { Comi, MD }\end{array}$ & $\begin{array}{l}\text { IRCCS San Raffaele } \\
\text { Scientific Institute, } \\
\text { Milan, Italy }\end{array}$ & $\begin{array}{l}\text { Analysis and interpretation of } \\
\text { the data, revising the } \\
\text { manuscript }\end{array}$ \\
\hline $\begin{array}{l}\text { Maria } \\
\text { Antonietta } \\
\text { Volonté, MD }\end{array}$ & $\begin{array}{l}\text { IRCCS San Raffaele } \\
\text { Scientific Institute, } \\
\text { Milan, Italy }\end{array}$ & $\begin{array}{l}\text { Clinical assessment, } \\
\text { analysis and } \\
\text { interpretation of } \\
\text { the data, revising } \\
\text { the manuscript }\end{array}$ \\
\hline $\begin{array}{l}\text { Massimo } \\
\text { Filippi, MD }\end{array}$ & $\begin{array}{l}\text { IRCCS San Raffaele } \\
\text { Scientific Institute } \\
\text { and Vita-Salute } \\
\text { San Raffaele } \\
\text { University, } \\
\text { Milan, Italy }\end{array}$ & $\begin{array}{l}\text { Study concept, analysis and } \\
\text { interpretation of the data, } \\
\text { revising the manuscript, study } \\
\text { supervisor }\end{array}$ \\
\hline
\end{tabular}

\section{Reference}

1. Hong B, Hermann EJ, Klein R, Krauss JK, Nakamura M. Surgical resection of osteolytic calvarial lesions: clinicopathological features. Clin Neurol Neurosurg 2010;112:865-869. 


\section{Neurology}

\section{Teaching NeuroImages: Osteolytic intraosseous meningioma causing transcalvarial herniation}

Aurora Semerano, Antonino Giordano, Mariarosa Terreni, et al.

Neurology 2020;95;e1110-e1111 Published Online before print July 10, 2020

DOI 10.1212/WNL.0000000000010091

\section{This information is current as of July 10, 2020}

\section{Updated Information \&} Services

References

Permissions \& Licensing

Reprints including high resolution figures, can be found at: http://n.neurology.org/content/95/8/e1110.full

This article cites 1 articles, 0 of which you can access for free at: http://n.neurology.org/content/95/8/e1110.full\#ref-list-1

Information about reproducing this article in parts (figures,tables) or in its entirety can be found online at:

http://www.neurology.org/about/about_the_journal\#permissions

Information about ordering reprints can be found online: http://n.neurology.org/subscribers/advertise

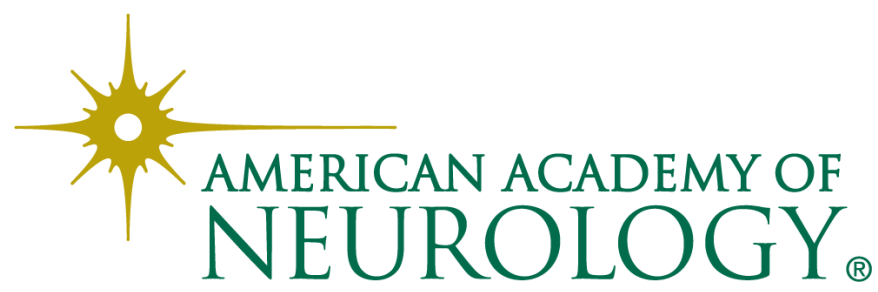

